\title{
Perú: un acercamiento al desempeño electoral en reelecciones subnacionales. Estudio cuantitativo de las elecciones provinciales de $2010^{+}$
}

\author{
DARÍO PEDRAGLIO TEILLIER* \\ Pontificia Universidad Católica del Perú \\ dario.pedraglio@pucp.edu.pe \\ https://doi.org/10.18800/rcpg.201602.003
}

\section{RESUMEN}

El presente trabajo se enfoca en discutir qué factores influyen en los resultados electorales que obtienen los alcaldes y/u organizaciones que se presentan a una reelección en el Perú - es decir, su desempeño electoral-, en particular en el nivel subnacional. Se analizan las elecciones provinciales del 2010 mediante una regresión lineal del porcentaje de votos que obtuvieron en sus intentos reelectorales. Utilizando una base de datos propia - construida a partir de información proveniente del Ministerio de Economía y Finanzas, Infogob y PNUD—, se encuentra que la capacidad de gasto de los incumbentes influye sobre el número de votos que estos obtienen; que el aumento del gasto en años electorales es visto favorablemente por los electores; que los candidatos que ganaron en 2006 con mayores porcentajes y mayor diferencia respecto al segundo se desempeńan mejor en las reelecciones; y que compartir partido con un candidato regional fuerte - es decir, que quedó entre los dos primeros de su elección- favorece al candidato del nivel inferior, en este caso, provincial. Reconociendo la posibilidad de que elementos distintos a los planteados importen, así como los límites de las hipótesis y variables esbozadas, el trabajo pretende, a partir de la información disponible y la revisión de distintas investigaciones, dilucidar elementos útiles para futuras aproximaciones respecto al comportamiento electoral en el nivel subnacional peruano.

Palabras clave: Comportamiento electoral, desempeño reelectoral, elecciones subnacionales, incumbentes, partidos políticos.

Peru: an approach to electoral performance in subnational reelections. Quantitative study of the 2010 provincial elections

\section{Abstract}

This paper aims to identify the determinants of incumbents' electoral performance on the 2010 Peruvian provincial elections. It uses data from several sources, from ministries to international organizations, to compile indicators at provincial level. Using a linear regression

\footnotetext{
* Licenciado en Ciencia Política y Gobierno por la Pontificia Universidad Católica del Perú.

${ }^{+}$Recibido el 8 de enero de 2017; aceptado el 19 de marzo de 2017.
} 
model to determine which factors influence vote percentages of the mayors and organizations who sought re-election, results showed that incumbents' expenditure capacity has an influence over their re-election result; voters have a favorable perception of an increase in expenditure in election years; incumbents who won the previous election with large numbers and by a greater margin tend to also perform well in re-election; and that sharing a political party with a strong regional candidate-first two places of his election-favors the provincial incumbent. The study's intention is to contribute, with the available information, to national literature on subnational electoral behavior.

Key words: Electoral behavior, electoral performance, subnational elections, incumbents, political parties. 


\section{INTRODUCCIÓN}

En el Perú, el comportamiento de los electores genera opiniones diversas ${ }^{1}$. En plenas elecciones municipales de 2014, Steven Levitsky, por ejemplo, sintetizaba la polémica en el diario La República:

Los peruanos no son estúpidos [...] lamentar la 'ignorancia', la 'falta de memoria' y hasta la estupidez del electorado peruano se ha vuelto una práctica común. El rey de los lamentos es Aldo Mariátegui, que saca del clóset su concepto del 'electarado' cada vez que se elige a quien no le gusta. Pero Mariátegui no está solo. Y el desprecio hacia el electorado peruano no se limita a la derecha. [...] muchos comentarios progresistas sobre los que votan por candidatos que 'roban pero hacen obras' revelan el mismo desdén [...] [E]n vez de despreciar al electorado peruano, sería mejor estudiar por qué la gente vota como vota. Como no ha habido mucha investigación sobre el comportamiento electoral peruano, sabemos muy poco (Levitsky, 2014).

Argumentos como los que describe el politólogo estadounidense se tornan comunes mientras más cerca se está de un proceso electoral. Cuando la contienda enciende discusiones, no es raro que el debate caiga en generalidades y prejuicios que terminan calando en la opinión pública. Qué mejor ejemplo que el concepto «electarado», acuñado por Aldo Mariátegui para describir a quien no comparte su posición política. Estas perspectivas, como señala Levitsky, no solo desdeñan un cierto comportamiento político, sino que impiden entender cómo deciden, políticamente, los ciudadanos; tema sobre el que «sabemos muy poco» (Levitsky, 2014).

Más allá de la elección en sí misma —o de juzgarla—, es importante analizar por qué las personas eligen a quienes eligen. La información obtenida de tal análisis debe generar conocimiento empírico sobre las razones que influyen en este tipo de decisiones, lo que permitirá que el debate académico se enriquezca y que los tomadores de decisiones cuenten con información al momento de formular y discutir políticas públicas. En particular, la necesidad de información es notoria en el ámbito subnacional; la mayoría de las investigaciones sobre comportamiento electoral en el Perú se centran en el nivel de gobierno nacional. La mayor parte de la información y el análisis no tiene relación con lo que ocurre en regiones, provincias ni distritos. Esto último trae consigo limitaciones importantes en el espacio académico y en el de políticas públicas: ¿es conveniente, por ejemplo, prohibir la reelección en el nivel subnacional? No es

\footnotetext{
1 En el plano académico, algunos le dan mayor importancia a la relación entre estructura social y voto; otros, al impacto de los clivajes y los atajos cognitivos; y otros, a la racionalidad de los electores (Sulmont, 2009). En el periodismo, como seńala Levitsky (2014), las opiniones también varían; por ejemplo, se explica el comportamiento político por el nivel educativo, la falta de memoria o el clientelismo.
} 
posible acercarse a una respuesta satisfactoria sin evidencia que ayude a un debate informado; y con la información que hay a la mano, es difícil pensar que tal discusión pueda darse.

El presente trabajo tiene el objetivo de contribuir a la información e investigación existente sobre el comportamiento electoral en el ámbito subnacional. En tal sentido, busca responder una pregunta bastante sencilla: ¿qué factores afectaron el desempeño electoral de los alcaldes y organizaciones provinciales que postularon a la reelección en las elecciones de 2010? Para responderla, se comenzó por revisar investigaciones sobre el fenómeno de la reelección —con énfasis en el ámbito subnacional - en casos como Estados Unidos, Colombia, España, Israel, Portugal, Chile y Brasil. Se identificó, asimismo, a todos los alcaldes y organizaciones provinciales que buscaron ser reelegidos en 2010 . Esto, con el fin de dilucidar qué elementos tomaron en cuenta los ciudadanos al momento de juzgar a sus autoridades.

Pero ¿por qué estudiar de manera particular esta performance electoral? Pues porque permite examinar dos ámbitos importantes del comportamiento electoral (Carlin, Singer y Zechmeister, 2015): los criterios que usan los ciudadanos al elegir a sus autoridades y al evaluar su desempeño, y el alcance de las elecciones como mecanismo de control ciudadano. Es, en tal sentido, una propuesta enfocada en los electores, con la intención de contribuir a conocer mejor cómo los ciudadanos determinan su voto y evalúan a las autoridades elegidas a nivel provincial.

Es importante recalcar que este trabajo no pretende introducirse en el debate respecto a la ventaja del incumbente. Para analizar tal fenómeno, el estudio debería contener no solo a quienes postulan a la reelección, sino también a sus opositores, mientras que aquí el objeto de estudio son solo aquellos alcaldes y/u organizaciones que, en las elecciones municipales provinciales de 2010, buscaron reelegirse.

Asimismo, la disponibilidad de datos y el diseño de la investigación limitan el análisis de otros elementos que, innegablemente, existen e importan. Por tal razón, las hipótesis y variables aquí planteadas están lejos de querer establecer preceptos excluyentes. Por el contrario, a partir de la información disponible y de la revisión de diversas investigaciones, se propone un acercamiento al poco estudiado asunto de cómo se decide el voto en el nivel subnacional, con la idea de colaborar con futuras aproximaciones sobre este tema. 


\section{REVISIÓN DE LITERATURA}

Como señalan Carlin, Singer y Zechmeister (2015), los sistemas políticos de los países de América Latina están en constante cambio; en la mayoría de elecciones el repertorio de actores y partidos varía constantemente. Lo mismo ocurre con el contenido de las plataformas que estos representan². Así, el hecho de que las opciones políticas en la región no sean ni coherentes ni duraderas tiene consecuencias en el comportamiento electoral; muchos ciudadanos no ven claramente qué distingue a cada partido, qué representa y propone cada cual, y ni siquiera qué diferenciaría a unos de otros en caso de ganar las elecciones:

Si estar políticamente informado y realizar un voto racional es una tarea compleja para ciudadanos en sistemas democráticos de largo aliento con pocas, y coherentes, opciones políticas [...], entonces la tarea del votante promedio latinoamericano es aún más complicada (Carlin, Singer y Zechmeister, 2015, p. 4).

Esta perspectiva es compartida por Muñoz (2010) para el caso peruano. La autora señala que en democracias como la peruana, los electores, debido a la dificultad de distinguir entre lo que representan y proponen los candidatos y las organizaciones, no definen su voto sobre la base de preferencias programáticas. En tal sentido, en el Perú se ha dado un proceso de desorientación cognitiva a partir de la crisis de los partidos tradicionales (Sulmont, 2009; Carrión, 1996). Por lo tanto, para acercarse al elector peruano es necesario poner énfasis en enfoques que se centren más bien en ciudadanos que: i) buscan establecer un vínculo con el Estado a través del intercambio de ventajas y/o bienes; ii) evalúan sus decisiones electorales según las expectativas redistributivas de los candidatos; y iii) prestan una mayor atención a las cualidades personales de los mismos (Muñoz, 2010):

En democracias relativamente nuevas, como la nuestra, suponer que todos los electores votan de acuerdo a preferencias programáticas puede constituir una limitación importante para comprender la complejidad de los mecanismos a través de los cuales sectores diversos del electorado deciden cómo votar (Muñoz, 2010, p. 44).

Si, como plantean estos autores, en el Perú lo programático no es lo más importante para los ciudadanos al momento de votar, entonces, ¿qué priorizan? A partir de la revisión de trabajos producidos en diversas partes del mundo que se han acercado al motivo central de esta investigación, y que contribuyen a entender al electorado, es posible reconocer cuatro elementos que podrían estar cumpliendo un papel en el comportamiento electoral subnacional en el Perú:

2 El escenario corresponde a la última década del siglo XX y lo que va del siglo XXI. 
i) el desempeño del incumbente; ii) las estrategias clientelares; iii) la fuerza electoral —que comprende el valor de competencia de los candidatos y la competitividad electoral de las provincias-; y iv) el arrastre. Cabe recalcar que algunos conceptos han sido reforzados por nociones planteadas por autores que no necesariamente se centran en la reelección o el comportamiento electoral. Estos, sin embargo, plantean percepciones valiosas respecto a cómo funciona la dinámica electoral. Es el caso, por ejemplo, de Zavaleta (2013), quien se centra en los políticos y en los candidatos, y no necesariamente en los electores.

Por su naturaleza, el desempeño, las estrategias clientelares y la fuerza electoral histórica de los incumbentes se relacionan con lo que Muñoz (2010) y Carlin, Singer y Zechmeister (2015) señalan como un posible factor influyente en la preferencia ciudadana: la percepción de los votantes respecto a la autoridad. Esto es, la labor del incumbente durante su mandato, sus características personales y la satisfacción de las expectativas. Desde este punto de vista, las elecciones sirven para que los votantes recompensen con su voto, o no, a las autoridades, según cómo califican su mandato y cómo perciben a los incumbentes o a sus organizaciones.

Key (1966) señala que el voto sirve como medio para castigar o premiar a un gobernante que pretende continuar ejerciendo sus funciones en un régimen democrático. Manin (1988) afirma que los votantes analizan los resultados obtenidos a lo largo del periodo de gobierno de un candidato que ya ejerció el poder para determinar si fueron negativos o positivos, y utilizan esta información para decidir su voto. No obstante, los factores tomados en cuenta por los electores pueden ser muchos y muy variados (Kitschelt y Altamirano, 2015). Estos van desde la competencia en la gestión — que puede dividirse en varios temas, como el presupuestal, el del crimen o el de los servicios públicos, por nombrar algunos-, la capacidad representativa y la identificación partidaria, hasta el carisma o la existencia de lazos clientelares (Kitschelt y Altamirano, 2015). Los elementos planteados en este análisis buscan acercarse a cuatro factores del abanico de variables.

\section{a. La performance del alcalde durante su gestión}

La idea es comprobar si buenas gestiones fiscales y mejoras en la calidad de vida de los electores impactan en la preferencia de estos últimos. Tal perspectiva se sustenta en la noción planteada desde la teoría de la rendición de cuentas vertical, que sostiene que las elecciones sirven como una oportunidad para que la ciudadanía evalúe a sus políticos, los haga responsables de sus éxitos o fracasos como autoridades, y los premie, o no, con su voto (Samuels y Hellwig, 2010; Sulmont, 2009). 
Tal premisa la han estudiado diversos autores, en varios países y contextos, y con resultados diversos: en ciertos casos hubo evidencia de una relación entre el desempeño de la autoridad y su performance reelectoral, mientras que en otros no se capturó tal relación ${ }^{3}$. A pesar de que los resultados de los trabajos sobre la rendición de cuentas electoral están inconclusos —o quizá por esto mismo—, tal relación podría estar dándose en el Perú; y aproximarse al caso peruano podría darle una mayor robustez al debate.

\section{b. La existencia de estrategias clientelares}

Kitschelt y Altamirano (2015) señalan que:

El intercambio clientelar es una de las muchas maneras en las que los votantes pueden conectarse con los políticos y obligar a que estos «rindan cuentas» [...] En muchos países, para la mayoría de los ciudadanos el clientelismo puede ser una alternativa para conseguir beneficios de un aparato gubernamental incapaz de idear o implementar políticas públicas. Esto no responde a los vínculos normativos propuestos por la teoría democrática y el sentido de justicia expresado por intelectuales y votantes educados. No obstante, el hecho de que agentes brindan estos servicios a cambio de votos es una realidad de la rendición de cuentas democrática (pp. 247 y 258).

Se estudian acá dos estrategias que podrían estar usando los alcaldes para aumentar su posibilidad de reelegirse. La primera es una variación del porcentaje de gasto público entre los años electorales y no electorales. La inclusión de un indicador que observe este aspecto responde a la necesidad de comprobar la existencia de ciclos en el gasto público —en los que los alcaldes con, entre otros, fines reeleccionistas aumentan el gasto público en los años electoralesy el posible impacto de estos sobre los resultados, algo que ha sido analizado en diversos países pero todavía no en el Perú.

Varios autores han estudiado esta relación desde distintos puntos de vista, para diferentes casos y con resultados tanto confirmatorios como de rechazo: Brun-Martos, Balaguer-Coll y Tortosa-Ausina (2014) encuentran el fenómeno en Espańa, así como un impacto electoral positivo del mismo; Brender y Drazen (2008) investigan — y confirman — su uso por parte de presidentes en distintos países del mundo, pero no hallan relación con los resultados de las elecciones;

\footnotetext{
3 Chubb (1988), Ebeid y Rodden (2006), Besley (2005), Cummins (2009), Cummins y Holyoke (2012) y Stone, Fulton, Maestas y Maisel (2010), para el caso estadounidense; Martins y Veiga (2013) y Sarpietro (2014) en Portugal; Brun-Martos et al. (2014) en España; Cassette, Farvaque, Héricourt y Jean (2009) y Cassette, Farvaque, Héricourt y Jean (2011) en Francia; Brender y Drazen (2008) en el nivel presidencial en distintos países del mundo; Sigalas (2011), quien se fija en el Parlamento Europeo; Brender (1999) en Israel; Pereira y Renno (2001), Rocha (2004), Litschig y Morrison (2012) y Sakurai y Menezes-Filho (2008) en Brasil; Eslava (2005) en Colombia; Abuelafia y Meloni (2000) en Argentina; Vergara (2014) en Chile; y Weyland (2001) para el Perú.
} 
Aidt, Veiga y Veiga (2011) descubren, para Portugal, que comportamientos oportunistas favorecerían las probabilidades de los incumbentes, en tanto que un mayor gasto en ańos electorales aumenta sus probabilidades, hallazgo que confirma lo encontrado por Veiga y Veiga (2007) para el mismo país; Brender (1999) no logra encontrar, para el caso israelí, relación entre la expansión de la política fiscal en años de elección y resultados de los candidatos; Sakurai y Menezes-Filho (2008) encuentran que en Brasil tal estrategia sí resulta beneficiosa para los incumbentes, por lo menos para el periodo estudiado; y Drazen y Eslava (2008) y Eslava (2005), para el caso colombiano, encuentran tanto este tipo de estrategias reelectorales como un impacto de las mismas en las preferencias de los electores4.

La segunda estrategia se refiere al uso de los empleos del sector público para ganar electores. Como señalan Kitschelt y Altamirano (2015), una de las formas en las que los políticos establecen vínculos clientelares con los ciudadanos — con el fin de ganar su voto a cambio de beneficios- es el acceso a empleos en el sector público. Esta premisa no ha sido tan estudiada como la del aumento del gasto en periodos cercanos a la elección; el único ejemplo, entre los casos revisados, es el de Cassette et al. (2009). Estos, si bien no se fijan solo en esta relación, encuentran que mayores gastos en personal estarían teniendo un impacto positivo en la reelección en las municipalidades francesas.

\section{c. La fuerza electoral histórica}

El tercer elemento planteado en este análisis es la fuerza electoral histórica, que puede dividirse en dos conceptos: el valor de competencia y la competitividad electoral. El valor de competencia, usado por Zavaleta (2013), se refiere a los valores de los candidatos, así como al impacto de las diferencias en las preferencias de los ciudadanos. Este valor de competencia, según Zavaleta (2013), estaría conformado por dos elementos: la «reputación personal» del candidato — sujeta a factores como el carisma- y los recursos materiales de los que este dispone. Ambos factores se traducen en una etiqueta pública —un nombre y un logo- que se comunica a los electores y que diferencia a un conjunto político de otro (Zavaleta, 2013). Esta es una idea también planteada

\footnotetext{
4 En el Perú, las autoridades tienen restricciones de gasto desde noventa días antes de los procesos electorales, lo que podría limitar su capacidad de concretar ciertas iniciativas. Además, es posible discutir la idea de que la finalización de obras en el último año responda necesariamente a una estrategia clientelar; no obstante, las investigaciones revisadas entienden que este fenómeno se da, por lo menos en cierta medida, debido al deseo reelectoral de las autoridades. Asimismo, el hecho de que tales aumentos se consideren estrategias clientelares no significa que los gastos no puedan satisfacer deseos y necesidades reales de la ciudadanía.
} 
por Berry, Berkman y Schneiderman (2000), quienes afirman que la fuerza electoral histórica de los incumbentes indica características que influyen en sus performances reelectorales. Esto porque los candidatos que, inicialmente, ganaron por márgenes pequeños pueden tener una escasez de simpatizantes o debilidades personales y/o materiales; problemas que, probablemente, se mantendrán en las elecciones para reelegirse 5 .

El segundo concepto de la fuerza electoral histórica es la competitividad electoral existente en la jurisdicción; en este caso, las provincias. Como señalan Carlin, Singer y Zechmeister (2015), la evaluación que hacen los electores se da en un contexto político — relacionado con la competitividad de la elección, entre otros factores- que influye en su decisión como votantes. Así, la fuerza electoral no solo muestra características de los incumbentes, relacionadas con su reputación y sus recursos materiales; también sirve para determinar la competitividad electoral en una jurisdicción, concepto también acuñado por Jacobson (1987), Garand (1991) y Sullivan y Uslaner (1978). Desde este punto de vista, los candidatos marginales — los que ganaron su primera elección por márgenes reducidos- deberían tener mayores dificultades para hacerse con la reelección. Estos candidatos se encuentran en una posición menos segura respecto a su continuidad en el cargo, factor que, según Brender (1999), también podría tener efecto sobre la gestión de la autoridad elegida.

\section{d. El arrastre electoral}

El cuarto elemento estudiado que podría injerir en el desempeño electoral de los incumbentes es el arrastre electoral: candidatos municipales que postulan a la elección junto con liderazgos fuertes en los niveles de gobierno superiores serían considerados por los votantes como opciones factibles (Chubb 1988; Berry, Berkman y Schneiderman, 2000). Este arrastre está relacionado con el ámbito institucional —llámense leyes, costumbres, tradiciones, etcétera- que, como sostiene Vergara (1997), influye también en las decisiones de las personas. Esto, porque en el Perú la ley electoral fuerza a que los comicios para los tres niveles de gobierno subnacionales (regional, provincial y distrital) sean el mismo día. El fenómeno — spill over, en inglés — ha sido comprobado en Estados Unidos por Berry et al. (2000), quienes señalan que los candidatos fuertes atraen más simpatizantes, y que, por ende, los candidatos del mismo partido en niveles de gobierno inferiores disfrutan de un spill over effect que

\footnotetext{
5 Relación estudiada, aunque en menor profundidad, por Cassetee et al. (2009), quienes se aproximan al tema en el caso francés; Aidt, Veiga y Veiga (2011) y Sarpietro (2014), para el portugués; y Brender (1999) para el israelí.
} 
mejora su chance electoral. Asimismo, Chubb (1988) sostiene que hay una fuerte influencia de los candidatos de niveles superiores sobre los resultados de las elecciones para las autoridades de niveles de gobierno inferiores.

Zavaleta plantea que algo similar ocurriría en Perú en el ámbito subnacional: «los candidatos municipales coaligados con un liderazgo regional que cuente con oportunidades de triunfo son considerados por los votantes como opciones factibles para ganar las elecciones locales» (2013, p. 22). Esta sería una costumbre entre los candidatos a cargos de niveles subnacionales, quienes buscarían aliarse con candidatos fuertes en niveles de gobierno superiores, para mejorar su performance en las elecciones. Una entrevista de Zavaleta al personero legal del Movimiento Regional Raíces, de Puno, ilustra la lógica que seguirían los políticos: «[...] todos [los candidatos] hacen una evaluación, y uno hace una autoevaluación y dice: "me interesa porque, al margen de que es un líder aquí, me interesa que me ayude porque arrastra electorado"» (Zavaleta, 2013, pp. 22-23). En tal sentido, esta parece ser una estrategia utilizada de manera sistemática por los candidatos a las municipalidades que vale la pena analizar, con la intención de comprobar su posible impacto en las preferencias del electorado.

Queda bastante claro que estos grupos de variables se introducen en el debate respecto a comportamientos de los electores peruanos que son planteados como posibles por Muñoz cuando sostiene que: «en democracias relativamente nuevas, como la nuestra, suponer que todos los electores votan de acuerdo a preferencias programáticas puede constituir una limitación importante para comprender la complejidad de los mecanismos a través de los cuales sectores diversos del electorado deciden como votar» (Muñoz, 2010, p. 44). Por esto, la intención es — centrando el foco del análisis en los electores- ampliar el abanico de posibles factores influyentes en el desempeño electoral de quienes buscan la reelección, con el fin de entender mejor cómo es que las personas estarían decidiendo su voto en contextos de desorientación política, tanto cognitiva como programática, fruto de la crisis partidaria existente en el país.

\section{Metodología}

En cuanto al método de trabajo, corresponde primero referirse a las hipótesis planteadas. Estas tienen la intención de brindar posibles respuestas respecto a qué factores toman en cuenta los ciudadanos al determinar su voto. Asimismo, lejos de cancelarse unas a otras, estas hipótesis se complementan y buscan ampliar el panorama que se tiene hasta el momento. 


\section{a. A mejor desempeño durante gestión, más opciones de reelegirse}

Como primera hipótesis, se trabaja la premisa de que los candidatos a la reelección con mayor competencia en la gestión de la municipalidad provincial tendrán una mejor performance electoral en sus intentos reelectorales. Concretamente, se plantea que: i) la variación en el Índice de Desarrollo Humano (IDH) y en el Índice de Densidad del Estado (IDE) — medidas de la calidad de vida y la capacidad del Estado, respectivamente- tendrá un efecto en las preferencias de los electores, es decir, sobre el desempeño reelectoral de los incumbentes ${ }^{6}$; y ii) que la capacidad de gasto tendrá, también, un efecto; mientras mejor sea esta, mejor le irá al alcalde en la reelección.

\section{b. Las estrategias clientelares aumentan los votos de los incumbentes}

La segunda hipótesis es que las estrategias clientelares utilizadas por los incumbentes tienen un efecto en la preferencia de los votantes. Así, se plantea que: i) habrá un aumento en el gasto en años electorales y este afectará las preferencias de los electores y, por lo tanto, aumentará el porcentaje de votos obtenido por el incumbente; y ii) el gasto en personal también afectará la performance: un mayor gasto en este rubro aumentará los votos obtenidos por el incumbente, dado que una forma de establecer vínculos clientelares es mediante el acceso a empleos en el sector público.

\section{c. A mayor fuerza electoral histórica, más posibilidades de reelección}

Se plantea que aquellos que ganaron con mayores márgenes de diferencia en las elecciones originales —es decir, aquellos con mayor fuerza electoral histórica - tendrán un mejor desempeño en la reelección. En tal sentido, la idea es que el valor de competencia de los incumbentes y la competitividad electoral existente en una provincia — las dos caras que componen el concepto de fuerza

\footnotetext{
6 Es importante seńalar dos limitaciones que plantea el uso del IDH y del IDE: a) ambos se construyen a partir de determinados ítems — como el acceso a servicios de salud, educación, vivienda y agua, y desagüe — que no se limitan a las competencias del gobierno provincial, sino que también responden a lo ejecutado por las autoridades de los demás niveles de gobierno; y b) aun cuando la variación corresponda a lo ejecutado por la autoridad provincial, es posible que los ciudadanos no le asignen a esta la responsabilidad del cambio. Sin embargo, ambas son limitaciones con las que se tiene que convivir, debido a la falta de mejores alternativas que sirvan como proxy para medir el desempeño de los alcaldes provinciales en lo que respecta a la calidad de vida de sus electores. Opciones como la Encuesta Nacional de Hogares no son representativas a nivel provincial (INEI, 2014), por lo que su uso comprometería por completo los resultados del trabajo.

7 Vale la pena señalar que existen importantes diferencias entre las provincias en cuanto a población, tamaño y dispersión de la ciudadanía en el territorio. Si bien el análisis toma en cuenta las diferencias en población de las provincias, es altamente probable que en provincias de territorio extenso y con población numerosa y/o dispersa, el uso de empleos públicos como herramienta clientelar sea limitado.
} 
electoral histórica- influirán en los votantes y, por ende, en la performance electoral de los incumbentes.

En relación con el valor, se espera que los incumbentes que en su primera elección obtuvieron altos porcentajes de votación tendrán un mejor desempeño electoral en la reelección. Por último, respecto a la competitividad, se plantea que candidatos marginales - aquellos que ganaron su primera elección por márgenes reducidos - deberían tener desempeños electorales menos fuertes en comparación con los que ganaron, inicialmente, por márgenes más amplios. Esto, porque habrá mayor competencia en su jurisdicción. Así, mientras mayor el porcentaje con el que ganó inicialmente el alcalde, y mayor el margen de victoria respecto al resto, mejor será su performance electoral en una posible reelección.

\section{d. A mayor arrastre del nivel superior, más votos para el incumbente}

La última hipótesis tiene que ver con la posible existencia, e impacto, del arrastre: la popularidad del candidato del mismo partido u organización que postula al nivel de gobierno superior — en este caso, el regional — tendrá un efecto sobre el porcentaje de votos obtenido por los incumbentes en las municipalidades provinciales. Esto es, los incumbentes que van en la misma organización que un candidato regional que quedó entre los dos primeros puestos tendrán un mejor desempeño en la reelección. Un candidato regional que queda en uno de los dos primeros lugares - y, por ende, pasa a la segunda vuelta, siempre y cuando el ganador de la primera elección no obtenga más del 50\%— puede ser considerado como fuerte, característica que ayudará al candidato del mismo partido en el nivel de gobierno provincial.

Vistas las cuatro hipótesis planteadas, corresponde explicar qué casos han sido estudiados, cómo se llevó a cabo el análisis estadístico y de qué manera se operacionalizaron las variables, tanto la dependiente como las independientes.

\subsection{Objeto de estudio, modelo estadístico y operacionalización de las variables}

El análisis toma en cuenta no solo los alcaldes incumbentes, sino también los partidos: si el alcalde no postuló a la reelección, pero su organización sí, se considera el resultado obtenido por esta. No incluir estos últimos casos, significaría dejar de lado información relevante respecto a la conducta del electorado.

Un ejercicio sirve para ilustrar lo recién dicho: dos alcaldes deciden no postular a la reelección; el primero porque, debido a que considera que tiene un caudal electoral suficiente, ha decidido postular a la presidencia regional; el segundo porque considera que su apoyo es sumamente bajo. En tal caso imaginado, es razonable pensar que, de haberse presentado, ambos alcaldes hubieran 
tenido performances distintas; sin embargo, si se fija la mirada en el resultado final, a saber, el hecho de que ninguno postuló, se equiparan casos desiguales y, por ende, se pierde información valiosa. En paralelo, si las organizaciones a las que pertenecen los alcaldes buscan ser reelegidas, será posible recoger las preferencias de los electores, que también reflejarán la percepción de los ciudadanos sobre lo hecho durante la gestión, sobre la fuerza electoral y sobre el arrastre. Así, se decidió incluir a las organizaciones para perder la menor cantidad de información posible, en tanto que las percepciones del electorado no solo afectan a la autoridad incumbente sino también al partido, y no «desaparecen» cuando el alcalde decide no reelegirse ${ }^{8}$.

Por otra parte, ¿por qué la selección de las elecciones de 2010? Porque para recoger la información se elaboró una base de datos propia a partir del recojo y la sistematización de estadística pertinente respecto a cada provincia en la que el alcalde o la organización de turno buscó la reelección, estadística que se halló disponible para el año en cuestión. Los datos sobre IDH e IDE provinieron del PNUD; los de capacidad de gasto, del Ministerio de Economía y Finanzas, gracias a su buscador amigable; y los relacionados con los resultados electorales, en la página web de Infogob.

En lo que respecta al método, por el número de casos se determinó el uso de una herramienta estadística para analizar la información recogida: un modelo de regresión lineal que permitió establecer si alguno de los factores planteados, o un grupo de estos, tuvo efecto sobre los votos obtenidos por los incumbentes. Siguiendo a Robert Nau (s/f), en modelos lineales la variable de interés, o dependiente, se predice de un número $k$ de variables explicativas usando una ecuación lineal. Si $y$ denota la variable dependiente, y $x_{1} \ldots x_{k}$ a las independientes, entonces se supone que el valor de $y$ en el tiempo $t$ se determina por la siguiente ecuación:

$$
y_{t}=B_{0}+B_{1} x_{1 t}+\ldots B_{k} x_{k t}+\varepsilon_{t}
$$

En donde $y_{t}$ es la variable dependiente — a saber, la performance electoral de los incumbentes-,$x_{k t}$ corresponde al conjunto de variables independientes, $B_{o}$ es el intercepto - el valor esperado de $y$ cuando todos los $y$ son cero-, $B_{k}$ es el coeficiente de las variables $x_{k t}$ y $\varepsilon_{t}$ es el error.

\footnotetext{
8 Hay unos pocos casos en los que alcaldes y organizaciones incumbentes compiten. Esto se da porque los primeros cambian de partido durante su mandato, y las organizaciones, por su parte, cambian de candidato. En tales casos se determinó utilizar la información referida a la autoridad y no al partido, dado que no era posible recoger ambas partes al mismo tiempo.
} 
Lo primero a tratar es la variable dependiente. En tanto que aquello que se quiere medir es la performance electoral de los incumbentes - tanto alcaldes como organizaciones-, esta es el porcentaje de votos válidos obtenido por estos en sus intentos reelectorales. Vista la variable dependiente, queda por operacionalizar las independientes. Con relación al desempeño del alcalde, se midió, en primer lugar, la capacidad de gasto, determinada por el promedio del porcentaje gastado respecto a lo presupuestado durante el periodo comprendido entre 2007 y 2010, así como el promedio per cápita del gasto total anual durante el total de la gestión.

En segundo lugar, se midió la variación en el IDH y del IDE, que busca medir cambios en las condiciones de vida de los ciudadanos y en el acceso de estos a servicios públicos. Esta variación se determinó mediante la tasa de cambio del IDH y del IDE entre 2007 y 2010:

$$
\left(\frac{I D H_{2007}-I D H_{2010}}{I D H_{2007}}\right) * 100
$$

Lo siguiente es tratar las posibles estrategias clientelares. La primera, relacionada al acceso a empleos públicos, se midió mediante un solo indicador: el promedio per cápita del total gastado durante la gestión en el rubro de salarios para personal. La segunda estrategia, relacionada a la existencia de un posible aumento del gasto en años electorales, se midió mediante una tasa de cambio, referida a los montos totales per cápita gastados. Se consideraron electorales el año de la elección y el año anterior — 2010 y 2009— y no electorales los dos primeros años de gestión — 2008 y 2007 - Estas tasas tomaron la siguiente forma, donde $g p$ se refiere al gasto público:

$$
\left[\frac{\{([g p 09+g p 10 \gamma 2)-([g p 07+g p 08]) 2\}}{([g p 07+g p 0872)}\right]{ }^{*} 100
$$

\footnotetext{
9 Otra alternativa hubiera sido usar una variable que midiera solo si los incumbentes ganaron —o no- su reelección. No obstante, esta forma "esconde» información sobre la performance electoral de los candidatos y las organizaciones, en tanto equipara desempeńos distintos. No es lo mismo, por ejemplo, obtener $25 \%$ de los votos y perder la elección, que perderla con 5\%; estos son resultados que, si bien pueden tener un final común — no ganar-, muestran diferencias respecto a las preferencias de los ciudadanos. Utilizar el porcentaje de votos obtenido por los incumbentes permite capturar esta información, lo que no ocurre si solo se toma en cuenta el resultado final.
} 
El siguiente grupo de variables se refiere a las relacionadas con la fuerza electoral histórica de los incumbentes, que sirvió para determinar tanto el valor de competencia como la competitividad electoral en la circunscripción. Para esta medición se usaron dos indicadores: a) el porcentaje de votos obtenido por el incumbente en las elecciones de 2006; y b) la diferencia respecto al segundo mejor votado en las mismas elecciones. Ambos indicadores permitieron observar cuánto valor tenía el incumbente, por sí solo, pero también recoger información respecto a cuánta competencia hubo en la contienda. La combinación de los elementos permitió observar el fenómeno no solo de manera absoluta, sino también relativa.

Dado que estas variables se correlacionaban, fue pertinente, antes de aplicar el modelo, realizar una prueba de componentes principales, para que ambos indicadores se unieran en una sola variable latente. Esto fue útil porque, al estar correlacionadas, si se introducían al modelo de manera independiente, se hubiera perdido la capacidad explicativa de ambos indicadores, dado que su información sería, hasta cierto punto, redundante. Con el índice creado —al que se llamó «fuerza electoral histórica»—, se recogió la capacidad explicativa de ambas y se solucionó el problema de la correlación.

Por último, la alternativa elegida para medir el arrastre consistió en crear una variable dicotómica. Se asignó la categoría de éxito $(y=1)$ a los incumbentes que compartían partido con los candidatos que lograron uno de los dos primeros puestos en las elecciones municipales regionales. La de fracaso $(\mathrm{y}=0)$ se asignó, en cambio, a los incumbentes de una organización distinta a los candidatos regionales que compitieron en la segunda vuelta de dicha elección.

El Cuadro 1 resume la información relacionada con las variables independientes.

Por la naturaleza cuantitativa de la investigación, los indicadores que conforman el modelo no buscan ser más que aproximaciones útiles para medir empíricamente factores y características señaladas como influyentes en la literatura sobre el comportamiento electoral. Esto con la intención de comprobar si tendrían un efecto o no. Asimismo, no fue posible incluir variables imposibles de recoger o de medir de manera tal que pudieran introducirse en un análisis estadístico; por ejemplo, el dinero gastado en la campaña, la cobertura mediática de cada candidato, la percepción directa de los ciudadanos respecto a la honestidad del incumbente, las ideologías de los electores o las percepciones de los clivajes sociales activados por los candidatos. 
Cuadro 1. Lista de variables independientes

\begin{tabular}{|c|c|c|}
\hline Variables independientes & Indicadores & Operacionalización \\
\hline \multirow[t]{2}{*}{$\begin{array}{l}\text { Desempeño electoral } \\
\text { durante gestión }\end{array}$} & Capacidad de gasto & $\begin{array}{l}\text { Promedio del porcentaje gastado (2007-2010) } \\
\text { Promedio gasto per cápita (2007-2010) }\end{array}$ \\
\hline & Variación IDH e IDE & $\begin{array}{l}\text { Tasa de cambio IDH (2007-2010) } \\
\text { Tasa de cambio IDE (2007-2010) }\end{array}$ \\
\hline \multirow[t]{2}{*}{ Estrategias clientelares } & Uso de empleos públicos & $\begin{array}{l}\text { Promedio gasto total per cápita salarios } \\
(2007-2010)\end{array}$ \\
\hline & $\begin{array}{l}\text { Aumento del gasto en } \\
\text { ańos electorales }\end{array}$ & $\begin{array}{l}\text { Tasa de cambio en el porcentaje gastado } \\
\text { Tasa de cambio gasto total }\end{array}$ \\
\hline \multirow[t]{2}{*}{ Fuerza electoral histórica } & Valor de competencia & Pocentaje de votos elecciones de 2006 \\
\hline & Competitividad electoral & $\begin{array}{l}\text { Diferencia respecto al segundo, elecciones de } \\
2006\end{array}$ \\
\hline Arrastre electoral & $\begin{array}{l}\text { Mismo partido que pasó } \\
\text { a } 2 \text { da vuelta en región }\end{array}$ & Variable dicotómica (éxito $=1$ y fracaso $=0$ ) \\
\hline
\end{tabular}

\section{ANÁLISIS DE LA INFORMACIÓN: ¿QUÉ DICEN LOS DATOS?}

Una primera aproximación respecto a la información recogida debe ser descriptiva. Esto con la intención de aclarar el panorama sobre los incumbentes y sus organizaciones; el gasto público municipal, tanto el referido al promedio como al total, a las variaciones de este durante la gestión y a cómo se distribuye; y los datos referidos a los índices de densidad estatal y de desarrollo humano, así como su variación durante los años de gobierno del incumbente.

Si se toman de manera conjunta los datos de las autoridades y organizaciones incumbentes, se observa que 170 de los $195(87,2 \%)$ se presentaron a la reelección ${ }^{10}$, y que resultaron reelectos $46(27,1 \%)$. El promedio de votos fue de $20,1 \%$, con una desviación de 10,6, lo que indicaría una alta heterogeneidad entre los resultados. Asimismo, 135 de estos 170 compitieron en las elecciones provinciales y regionales, con un promedio de 15,3\% en estas últimas; 50 (36\%) de estos candidatos en el nivel de gobierno superior resultaron en uno de los

\footnotetext{
10 Este número (170), en comparación con el de alcaldes que postularon a la reelección (126), indica que 44 organizaciones buscaron la reelección aun cuando el alcalde no lo hizo.
} 
dos primeros lugares en su elección. Cabe señalar que en 2007, momento en el que alcaldes y organizaciones incumbentes ganaron originalmente la municipalidad, lo hicieron con un promedio del 30\%. Además, el margen de victoria fue de casi $8 \%$ frente a su más cercano opositor, en tanto que este último obtuvo, en promedio, 22,4\%.

Si bien las tasas efectivas de reelección no son especialmente altas $(27,8 \%$, $26,3 \%$ y $27,1 \%$, respectivamente), que los porcentajes de votos obtenidos por autoridades y organizaciones incumbentes sean heterogéneos indica que hubo diferencias importantes en los desempeños reelectorales, a pesar del resultado final común. Dicho de otra manera, si bien muchos perdieron, las derrotas sucedieron de formas distintas. Esto hablaría de importantes diferencias entre los incumbentes, que no se estarían manifestando en una reelección efectiva pero que, no obstante, vale la pena estudiar, pues darán indicios respecto a qué toman en cuenta los ciudadanos al momento de determinar su voto y cómo es que el contexto podría estar influyendo.

Cuadro 2. Cuadro descriptivo de alcaldes y organizaciones incumbentes

\begin{tabular}{|l|c|c|c|c|}
\hline & No & $\%$ & $\#$ & Desv. estándar \\
\hline Postularon reelección & 195 & $\mathbf{8 7 , 2 \%}$ & 170 & \\
\hline Exitosos & 170 & $27,1 \%$ & 46 & \\
\hline Porcentaje de votos & 170 & $20,1 \%$ & $\#$ & 10,6 \\
\hline $\begin{array}{l}\text { Postuló solo organización incumbente } \\
\text { (alcalde no) }\end{array}$ & 170 & $25,9 \%$ & 44 & \\
\hline Elección regional & 170 & $79,4 \%$ & 135 & \\
\hline Segunda vuelta & 135 & $36,0 \%$ & 50 & \\
\hline Porcentaje de votos & 135 & $15,3 \%$ & & 12,24 \\
\hline
\end{tabular}

En cuanto al gasto, entre 2007 y 2010 las municipalidades desembolsaron, en promedio, $70,2 \%$ de lo que originalmente presupuestaron, aunque con una desviación alta —a saber, 12.4- lo que indicaría una considerable variación entre los casos. El dinero promedio gastado por las municipalidades provinciales entre estos años fue de $S / 35500000$, con una desviación estándar, una vez más, bastante alta. En términos per cápita, esto se traduce en un promedio de 279 soles por persona, cifra que, sin embargo, varía mucho entre las observaciones. Resulta relevante señalar que 65,8\% del total gastado en promedio por las municipalidades provinciales fue asignado al gasto de capital, largamente el rubro de mayor asignación presupuestal. En promedio, se gastó 66,6\% de lo 
presupuestado en el rubro de gasto de capital, en comparación con el 89,3\% para pago de salarios de personal municipal, aunque el monto asignado a este rubro solo correspondió a un $10 \%$ del total gastado.

Por otro lado, hubo un aumento de $18,4 \%$ en la capacidad de gasto en los ańos considerados como electorales (2010 y 2009) respecto a los no electorales (2008 y 2007), así como un aumento de $73,3 \%$ en el total gastado. Es decir, hubo no solo una mayor capacidad de gasto, sino que en los ańos electorales se gastó casi el doble que en los no electorales. Estos resultados son un indicio de que, como se planteó en las hipótesis, incumbentes estarían gestionando el presupuesto estratégicamente, probablemente con miras a una reelección. Por último, en relación con el IDE y el IDH, el primero subió 13,9\% en los años de gestión, es decir, entre 2007 y 2010; y el segundo aumentó, en promedio, 21,3\%. Ambos resultados están acompañados de desviaciones estándar bajas, por lo que los datos obtenidos de las observaciones son relativamente homogéneos.

Con el panorama descriptivo expuesto, es posible pasar a presentar los resultados encontrados a partir del modelo estadístico. Las variables explicativas introducidas responden a las hipótesis planteadas previamente, con el fin de analizar si las nociones planteadas eran correctas o no.

\subsection{Análisis estadístico: ¿qué resultados se encuentran?}

A continuación se presentarán los resultados del modelo estadístico planteado. Este contempla dos variables de control: el ingreso por canon y el ingreso por donaciones, ambos calculados respecto al total gastado por la municipalidad. La variable dependiente es el porcentaje de votos obtenido por alcaldes y organizaciones que postularon a la reelección en las elecciones municipales provinciales de 2010. Las variables explicativas son: i) la presencia, o no, del candidato regional del mismo partido en la segunda vuelta; ii) la fuerza electoral histórica; iii) el promedio del porcentaje ejecutado respecto al presupuestado; iv) el gasto total per cápita; v) la variación del IDH y del IDE; vi) la variación entre años electorales y no electorales del gasto total per cápita; y vii) el gasto en personal per cápita.

En el Cuadro 3 se observa que el modelo estaría siendo explicativo, en tanto que hay un efecto de distintas variables planteadas sobre la votación obtenida por los incumbentes. 
Cuadro 3. Resultado alcaldes y organizaciones

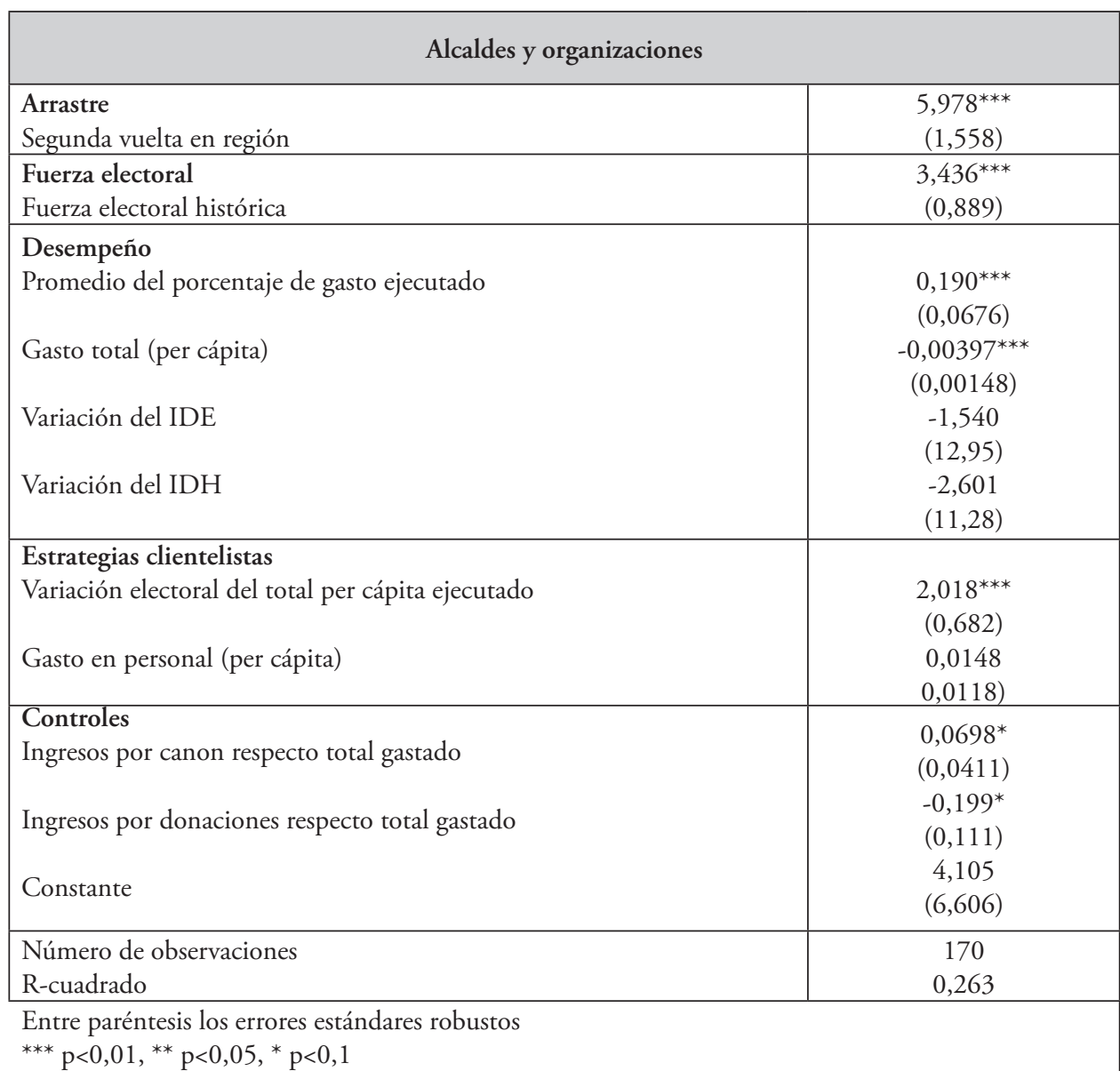

En primer lugar, hay un efecto positivo de la fuerza electoral del candidato de la misma organización en el nivel de gobierno superior sobre la performance electoral en la reelección. Así, compartir partido con quien quedó en uno de los dos primeros puestos en las elecciones regionales aumenta el número de votos obtenido por los incumbentes provinciales. Manteniendo el resto de indicadores constantes, el hecho de que los incumbentes participen en las elecciones en el mismo partido que un candidato regional que quedó primero o segundo, significa un aumento de casi seis puntos porcentuales en los votos obtenidos en un posible intento reelectoral. De esta manera, si a dos candidatos a la reelección -A1 y A2, por ejemplo- solo los distingue el hecho de pertenecer, o no, al mismo partido que un candidato regional fuerte —a saber, que pasó a segunda vuelta—, esa sola diferencia significará para A1 —el que compitió 
junto al candidato regional fuerte- una ventaja de seis puntos respecto a A2 en sus respectivos resultados.

En segundo lugar, la fuerza electoral histórica también estaría afectando positivamente los porcentajes de votos obtenidos por alcaldes y organizaciones que buscan la reelección. En tal sentido, incumbentes inicialmente más preponderantes en términos electorales tendrían mejores rendimientos en las reelecciones. Por cada unidad que aumente el índice de la fuerza electoral histórica — cuya media se sitúa en cero- se puede esperar un aumento de más de tres puntos porcentuales en los votos obtenidos por los incumbentes en la reelección, siempre y cuando el resto de factores se mantengan constantes. Usando el mismo ejemplo de antes, si los incumbentes A1 y A2 solo se diferencian en la fuerza electoral histórica de cada uno — siendo la de A1 una unidad superior a la de A2-y los demás elementos no cambian, entonces el primero obtendrá una votación casi cuatro puntos más alta que la que conseguirá el segundo incumbente.

En tercer lugar, se ve, en relación con el modelo 1, que existe un impacto positivo de la eficacia en el gasto ejecutado — respecto al presupuestado— sobre el desempeño electoral de los incumbentes; es decir, una mayor capacidad en este rubro aumenta los votos de los candidatos a la reelección. Así, en los casos analizados, es posible afirmar que, de mantenerse todo lo demás constante, aumentar en una unidad el promedio ejecutado durante la gestión traería consigo un incremento de 0,2 en el porcentaje de votos obtenido en la reelección. Siguiendo la lógica de los ejemplos planteados, si a los incumbentes A1 y A2 los diferencia solo su capacidad de ejecutar el gasto —en el caso del primero el promedio de esta fue de $71 \%$, mientras que en el del segundo fue de $61 \%$ - la diferencia de diez unidades entre uno y otro se traduciría en una ventaja de dos puntos en la votación reelectoral del primero, en relación con la del segundo.

En cuarto lugar, el gasto total per cápita estaría teniendo un efecto pero, a diferencia de lo que ocurría con los demás factores, este sería negativo: mayores gastos totales significan una menor votación en la reelección, de mantenerse sin cambios el resto de factores. Sin embargo, el efecto negativo que tendría el gasto total sobre el desempeño en las reelecciones es de poca magnitud, dado que la intensidad de este es muy baja. Esto porque el aumento de una unidad en el total gastado por persona significa una disminución de solamente poco menos de 0,004 en el porcentaje de votos obtenido por los incumbentes. Así, por ejemplo, un incumbente que gasta 1500 soles per cápita más que otro, manteniendo los demás factores constantes, obtendrá una votación solamente un punto menor que la del otro. Esto hace que, para que haya un efecto considerable en las 
preferencias de los ciudadanos, la diferencia tendría que ser considerable, lo que resulta difícil teniendo en cuenta que el promedio gastado por persona, en los casos observados, es de 279 soles.

Por último, se observa un impacto positivo de la variación en el total gastado entre ańos electorales y no electorales. Esto indica que el aumento en el gasto en los años más cercanos a los comicios estaría siendo tomado en cuenta, favorablemente, por los votantes. Para los casos analizados, la intensidad del efecto positivo que significa el aumento del gasto en los ańos electorales se traduce - si se mantienen sin cambios el resto de factores - en un incremento de poco más de dos puntos en el porcentaje de votos obtenidos en la reelección por cada unidad que sube el gasto per cápita total en los años electorales. En tal sentido, si la tasa de crecimiento del gasto total per cápita — cuyo promedio es poco más de cero- de un incumbente (A1) es un punto mayor que la de otro (A2), pero el resto de factores se mantienen constantes, se puede esperar que el incumbente que aumentó más su gasto (A1) en los años electorales tendrá dos puntos más en el porcentaje de votos en su intento reelectoral.

\section{Conclusiones}

Corresponde ahora contrastar los resultados descritos con las respuestas tentativas planteadas al inicio, para analizar cómo los conceptos planteados se relacionan con las preferencias de los electores y discutir el caso peruano en relación con las evidencias encontradas en las demás experiencias revisadas.

a) Respecto a la primera hipótesis — que planteaba una relación entre el desempeño en la gestión y resultados electorales en la reelección-, los resultados indican que los electores sí estarían fijándose en el desempeño de sus autoridades al momento de decidir sus votos. Como se planteaba, habría un uso de las elecciones como un mecanismo de rendición de cuentas por parte del electorado, que se fija en el desempeño de sus autoridades elegidas en lo que se refiere a la capacidad de gasto y al total que estas gastaron. Tal resultado sitúa al Perú entre los casos en los que hay evidencia de la existencia de una rendición de cuentas vertical, en tanto que los electores estarían fijándose en el desempeño pasado de sus autoridades para determinar sus preferencias electorales ${ }^{11}$.

\footnotetext{
11 Otros países en los que se encuentra evidencia similar: Estados Unidos (Besley, 2005; Cummins y Holyoke, 2012; Cummins, 2009; Trounstine, 2009; Ebeid y Rodden, 2006; y Alt y Lowry, 2010), España (Brun-Martos et al., 2014), Francia (Cassette et al., 2009 y Cassette, Farvaque y Héricourt, 2011); Portugal (Martins y Veiga, 2013; y Sarpietro 2014), Israel (Brender 1999), Brasil (Litschig y Morrison, 2012; Sakurai y Menezes-Filho ,2008; Rocha, 2004; y Pereira y Renno, 2001) y Chile (Vergara, 2014).
} 
Existe evidencia, entonces, de que hay un efecto positivo de la capacidad de gasto, lo que podría indicar que los electores están favoreciendo a autoridades con mejor performance en este rubro durante su mandato. Sin embargo, contrario a lo que se planteó como hipótesis, los electores no solo no estarían prefiriendo un mayor gasto total, sino que, más bien, hay indicios de que esto es percibido negativamente por la ciudadanía. Vistos ambos resultados en conjunto, la evidencia indicaría que los votantes estarían prefiriendo a autoridades que ejecutan lo que comprometieron presupuestalmente, pero evalúan de manera negativa a las autoridades que incurren en mayores gastos per cápita totales. Este efecto negativo, no obstante, es bastante pequeño, por lo que tendrían que ser sumas considerables para generar variaciones significativas en el porcentaje de votos obtenido.

b) En relación con el impacto de estrategias clientelares, los resultados indican, en primer lugar, que no es posible afirmar que mayores gastos en personal —aun controlado por la población - afecten positivamente los resultados electorales en la reelección ${ }^{12}$. En cambio, sí es posible afirmar que: hubo un aumento tanto del gasto total como de la capacidad de gasto entre años electorales y no electorales; y que hay evidencia a favor de la hipótesis planteada de que los aumentos en el gasto total — per cápita— en años electorales son tomados en cuenta por los electores al evaluar a sus autoridades y decidir sus $\operatorname{votos}^{13}$.

c) Respecto a la fuerza electoral histórica, los resultados arrojan indicios a favor de la hipótesis de que esta tendría un efecto sobre las preferencias de los votantes. Hay, entonces, evidencia a favor de la importancia de la fuerza electoral histórica ${ }^{14}$, lo que indica que habría características que se mantienen estables en el tiempo — relacionadas con el valor de competencia de incumbentes y la competitividad electoral de la jurisdicción- que influyen en las preferencias de los electores.

En tal sentido, en primer lugar, hay evidencia que favorece la idea de que hay elementos que se mantendrán a futuro — ligados a características personales, a una escasez de simpatizantes y/o de recursos - y que estarían causando diferencias en las preferencias de los votantes, lo que tiene un efecto en las

\footnotetext{
12 Esta es evidencia distinta a la encontrada por Cassette et al. (2009) para el caso francés.

13 Estos son resultados que dan indicios similares a los encontrados en España (Brun-Martos et al., 2014), Portugal (Aidt, Veiga y Veiga 2011; y Veiga y Veiga 2007), Brasil (Sakurai y Menezes-Filho, 2008) y Colombia (Drazen y Eslava 2008; y Eslava 2005).

14 Como encuentran Berry, Berkman y Schneiderman (2000), para el caso estadounidense, Cassette et al. (2009) en Francia, y Aidt, Veiga y Veiga (2011) y Sarpietro (2014) para Portugal.
} 
performances electorales de los incumbentes (Berry, Berkman y Schneiderman, 2000; y Zavaleta, 2013). En segundo lugar, la evidencia resultante iría acorde a la idea de que la competitividad electoral tendría un rol en cómo determinan los ciudadanos sus votos. Como sostienen Carlin, Singer y Zechmeister (2015), pareciera que la evaluación que hacen los electores de los elementos que toman en cuenta al elegir a sus autoridades se da en un contexto - relacionado con la competitividad de la elección, entre otros factores- que influye en las decisiones de los votantes. Así, de forma similar a lo que señalan Jacobson (1987), Garand (1991) y Sullivan y Uslander (1978), la evidencia encontrada en este análisis favorece la idea de que hay circunscripciones que presentan contextos más competitivos y menos estables, lo que termina influenciando cómo los votantes determinan sus preferencias.

d) Por último, los resultados evidencian la existencia del arrastre electoral como un factor que condiciona la evaluación de los ciudadanos al momento de decidir su voto. Como indica Zavaleta (2013), los candidatos de niveles de gobierno inferiores — en este caso, provinciales — tienen mejores desempeños en elecciones cuando compiten ligados con liderazgos regionales fuertes. Tal característica indicaría que los electores ven como opciones más factibles para ser reelegidos a los incumbentes que compiten en alianza con candidatos regionales fuertes.

Así, como sostienen Chubb (1988) y Berry, Berkman y Schneiderman (2000) para el caso de Estados Unidos, en el Perú también ocurriría que una mayor fuerza de los candidatos en niveles superiores de gobierno atraería a votantes hacia los candidatos del mismo partido en niveles de gobierno inferiores. En el caso peruano, es muy probable que la configuración de las reglas de juego sea un factor importante para entender el porqué de este fenómeno. Esto por una razón sencilla: las elecciones para el nivel de gobierno regional se dan el mismo día que las del nivel de gobierno. Tal diseño, muy probablemente, está condicionando la evaluación y la elección de los votantes, y favoreciendo a los candidatos que compiten con una alternativa regional fuerte.

Lo manifestado en este trabajo no significa que los electores no estén siendo influidos por elementos que no se tomaron en cuenta, dada la disponibilidad de información y considerando el diseño de la investigación. En este sentido, es posible que ciertos clivajes (políticos, sociales, económicos o ideológicos) sean parte de lo que los electores toman en cuenta. Del mismo modo, a pesar de la crisis partidaria, quizás haya un interés programático por parte de la ciudadanía. La intención no es negar la posibilidad de que estos elementos importen, sino procurar un acercamiento que, con la información disponible, 
brinde luces respecto a cómo las personas terminan por decidir su voto en el nivel subnacional. La idea, entonces, no es que esta sea la respuesta definitiva a las preguntas planteadas al iniciar la investigación, sino, en cambio, trazar un camino que abra trocha para futuras aproximaciones respecto al comportamiento electoral en el nivel subnacional peruano.

Vistos de manera conjunta, los resultados aportan evidencia que favorece la idea de que los votantes estarían tomando en cuenta la labor y las características de las autoridades y organizaciones electas al momento de decidir su voto. Los hallazgos, en tal sentido, favorecen la hipótesis de que los ciudadanos estarían usando los procesos electorales para evaluar a las autoridades elegidas cuatro años antes. Esta evaluación de los votantes giraría en torno a la capacidad de gestión presupuestal, a la existencia de estrategias clientelares —en particular, el aumento del gasto en años electorales- y al valor de competencia de los incumbentes. Tales son hallazgos acordes a la idea de que los votantes estarían fijándose en los desempeños pasados y utilizando las elecciones como herramienta de rendición de cuentas para/con las autoridades elegidas.

No obstante, los resultados del presente trabajo aportan evidencia de que esta evaluación estaría influida por elementos que no se relacionan con el desempeño de los incumbentes durante sus mandatos. Esto, porque la competitividad electoral también estaría influyendo en cómo deciden su voto los electores, así como el hecho de que los incumbentes compitan junto con liderazgos regionales fuertes. Dicho de otra manera, los resultados aportan indicios de que los electores no se fijan solamente en cómo gestionó el alcalde el presupuesto que tuvo durante su mandato; también toman en cuenta la fuerza del incumbente en relación con la de las demás candidaturas existentes, así como el hecho de que los candidatos a la reelección compartan organización con candidatos regionales fuertes.

Por último, estos resultados muestran que —en tanto que hay evidencia de que los ciudadanos utilizan las elecciones como un mecanismo para obligar a que las autoridades rindan cuentas - la prohibición de la reelección en el nivel subnacional podría estar quitándoles a los electores una herramienta que están usando para castigar o premiar a los incumbentes. Más allá de si gustan o no los criterios usados por los ciudadanos, vale la pena considerar si la prohibición realmente favorece al sistema democrático o si, más bien, se le está quitando a la ciudadanía un mecanismo útil para mantener accountable a las autoridades subnacionales, especialmente tomando en cuenta que la evidencia parece indicar que la reelección sí está siendo utilizada en este sentido por los ciudadanos. 


\section{BibLIOGRAFÍA}

Abuelafia, E. y Meloni, O. (2000). Determinantes económicos de los resultados electorales en Argentina: Evidencia a partir de datos panel. Tucumán, Argentina: Universidad Nacional de Tucumán.

Aidt, T., Veiga, F. y Veiga, L. (2011). Election results and opportunistic policies: A new test of the rational political business cycle model. Public Choice, 148(1-2), 21-44, doi: 10.1007/ s11127-010-9644-3

Alt, J. y Lowry, R. (2010). Transparency and accountability: Empirical results for US states. Journal of Theoretical Politics, 22(4), 379-406, doi: 10.1177/0951629810375641

Aragón, J. e Incio, J. L. (2014). La reelección de autoridades regionales y municipales en el Perú, 2006-2014. Argumentos, 8(5), 16-30.

Balaguer-Coll, I., Brun-Martos, M. T., Forte, A. y Tortosa-Ausina, E. (2014). Determinants of local governments' reelection: new evidence based on a Bayesian approach (documento de trabajo). Castellón de la Plana, España: Universidad Jaume I.

Berry, W., Berkman, M. y Schneiderman, S. (2000). Legislative Professionalism and Incumbent Reelection: The Development of Institutional Boundaries. The American Political Science Review, 94(4), 859-874, doi: 10.2307/2586212

Besley, T. (2005). Principled Agents? The Political Economy of Good Government. Londres, Reino Unido: Oxford University Press.

Brender, A. (1999). The Effect of Fiscal Perfomance on Local Elections Results in Israel: 1989-1998 (documento de trabajo 99.04). Jerusalén, Israel: Departamento de Investigación del Banco de Israel.

Brender, A. y Drazen, A. (2008). How do Budget Deficits and Economic Growth Affect Reelection Prospects? Evidence from a Large Panel of Countries. The American Economic Review, 98(5), 2203-2220, doi: 10.1257/aer.98.5.2203

Brun-Martos, I. y Balaguer-Coll, T. (2013a). Política presupuestaria y elecciones. Auditoría Pública, $60,65-72$.

Brun-Martos, I. y Balaguer-Coll, T. (2013b). El efecto del gasto público sobre las posibilidades de reelección de los gobiernos locales. Revista de Contabilidad, 16(1), 74-80, doi: 10.1016/ s1138-4891(13)70008-6

Carlin, R., Singer, M. y Zechmeister, E. (eds.) (2015). The Latin American Voter. Pursuing representation and accountability in challenging contexts. Ann Arbor, MI: University of Michigan Press, doi:10.3998/mpub.8402589

Carrión, J. (1996). La opinión pública bajo el primer gobierno de Fujimori: ¿de identidades a intereses? En F. Tuesta (ed.), Los enigmas del poder: Fujimori 1990-1996 (pp. 277-302). Lima, Perú: Fundación Friedrich Ebert.

Cassette, A., Farvaque, E. y Héricourt, J. (2011). Two-round elections, one-round determinants? Evidence from the French municipal elections. Public Choice 156(3-4), 563-591, doi: 10.1007/ s11127-012-9913-4

Cassette, A., Farvaque, E., Héricourt, J. y Jean, N. (2009). Budget structure and reelection prospects: Empirical evidence from french local elections (documento de trabajo). Bonn, Alemania: Zentrum für Europäische Integrationsforschung (ZEI).

Chubb, J. (1988). Institutions, the Economy, and the Dynamics of State Elections. The American Political Science Review, 82(1), 133-154, doi: 10.2307/1958062

Córdova, B. e Incio, J. L. (2013). La ventaja del incumbente en el ámbito subnacional: un análisis de las dos últimas elecciones municipales en el Perú. Papel Político, 18(2), 415-436.

Cox, G.y Morganstern, S. (1988). The Incumbency Advantage in Multimember Districts: Evidence from the U.S. States. Legislatives Studies Quarterly, 20(3), 329-349. 
Cummins, J. (2009). Issue Voting and Crime in Gubernatorial Elections. Social Science Quarterly, 90(3), 632-651, doi: 10.1111/j.1540-6237.2009.00635.x

Cummins, J. y Holyoke, T. (2012). Electoral Accountability and Fiscal Policy in the U.S. States: A Reassessment. Department of Political Science. Fresno, CA: California State University.

Dahl, R. (2008). La igualdad política. Buenos Aires, Argentina: Fondo de Cultura Económica.

Drazen, A. y Eslava, M. (2008). Electoral manipulation via Voter-Friendly Spending: Theory and Evidence (documento de trabajo). Bogotá, Colombia: Universidad de los Andes.

Ebeid, M. y Rodden, J. (2006). Economic Geography and Economic Voting: Evidence from the U. S. States. British Journal of Political Science, 36(3), 527-547, doi: 10.1017/S0007123406000275

Eslava, M. (2005). Political budget cycles or voters as fiscal conservatives? Evidence from Colombia. Bogotá, Colombia: Universidad de los Andes-CEDE.

Friedman, J. y Holden, R. (2009). The Rising Incumbent Reelection Rate: What's Gerrymandering Got to Do With It? The Journal of Politics, 71(2), 593-611, doi: 10.1017/s0022381609090483

Garand, J. (1991). Electoral Marginality in State Legislative Elections, 1968-86. Legislative Studies Quarterly, 16(1), 7-28, doi: 10.2307/439964

INEI-Instituto Nacional de Estadística e Informática (2014). Ficha técnica. Encuesta Nacional de Hogares. Lima, Perú: INEI.

Jacobson, G. (1987). The Marginals Never Vanished: Incumbency and Competition in the U.S. House of Representatives, 1952-2. American Journal of Political Science, 31(1), 126-141, doi: $10.2307 / 2111327$

Key, V. O. (1966). The Responsible Electorate: Rationality in Presidential Voting. Cambridge, MA: Harvard University Press. doi: 10.4159/harvard.9780674497764

Kitschelt, H. y Altamirano, M (2015). Clientelism in Latin America: Effort and Effectiveness. En R. Carlin, M. Singer y E. Zechmaister (eds.), The Latin American Voter. Pursuing representation and accountability in challenging contexts (pp. 246-274). Ann Arbor, Estados Unidos: University of Michigan Press.

Levitsky, S. (5 de octubre de 2014). Elecciones y tarados. Diario La República. Recuperado de http://larepublica.pe/columnistas/aproximaciones/elecciones-y-tarados-05-10-2014

Litschig, S. y Morrison, K. (2012). Government Spending and Reelection: Quasi Experimental Evidence from Brazilian Municipalities. (Documento de trabajo no 515). Barcelona, Espańa: GSE Working Paper Series..

Manin, B. (1988). Los principios del gobierno representativo. Madrid, España: Alianza.

Martins, R. y Veiga, F. J. (2013). Economic voting in Portuguese municipal elections. Public Choice, 155(3), 317-334, doi: 10.1007/s11127-011-9849-0

Milyo, J. y Groseclose, T. (1999). The Electoral Effects of Incumbent Wealth. Journal of Law and Economics, 42(2), 699-722. doi: 10.1086/467439

Muñoz, P. (2010). Análisis de resultados del perfil del elector peruano. En Perfil del elector peruano (pp. 37-49). Lima, Perú: Jurado Nacional de Elecciones.

Nau, R. (s/f). Notes on linear regression analysis. Recuperado de http://people.duke.edu/ -rnau/ notes_on_linear_regression_analysis--robert_nau.pdf.

Pereira, C. y Renno, L. (2001). Successful re-election strategies in Brazil: the electoral impact of distinct institutional incentives. Electoral Studies del Centro de Estudios Brasileros de la Universidad de Oxford, 22(3), 425-448, doi.org/10.1016/S0261-3794(01)00057-9

Rocha, A. (2004). Mayoral fiscal performance and reelection succes. Washington D.C.: Institute of Brazilian Business and Public Management Issues, George Washington University.

Sakurai, S.N. y Menezes-Filho, N. (2008). Fiscal policy and reelection in Brazilian municipalities. (Documento de trabajo del Departamento de Economía de la Universidad de Sao Pablo). São Paulo, Brasil: Universidad de São Paulo. 
Samuels, D y Timothy H (2010). Electoral Accountability: A Conceptual and Empirical Reassessment. (Versión preliminar). Recuperado de http://www.polisci.umn.edu/-dsamuels/documents/ Hellwig-SamuelsAPSA2008.pdf.

Sarpietro, S. (2014). Determinants of reelection in Portuguese municipal elections. Roma, Italia: Universidad Internacional del Estudio Social.

Sigalas, E. (marzo de 2011). Does Performance Matter? MEP Activity Levels and Re-Election. En Conferencia Bienal de la European Union Studies Association (EUSA). Boston, Estados Unidos.

Stone, W, Fulton, S., Maestas C. y Maisel, L. (2010). Incumbency Reconsidered: Prospects, Strategy Retirement, and Incumbency Quality in the U.S. House Elections. The Journal of Politics, 72(1), 178-190, doi: 10.1017/S0022381609990557

Sullivan, J y E Uslaner (1978). Congressional Behavior and Electoral Marginality. American Journal of Political Science, 22(3), 536-553, doi: 10.2307/2110460

Sulmont, D. (2009). Una revisión de literatura de los estudios sobre el comportamiento electoral en el Perú. Lima, Perú: Escuela de Gobierno y Políticas Públicas, Pontificia Universidad Católica del Perú.

Sulmont, D. (2010). Los enfoques teóricos sobre el comportamiento electoral. Lima, Perú: Pontificia Universidad Católica del Perú.

Trounstine, J. (2009). Information, Turnout, and Incumbency in Local Elections (manuscrito). Princeton, Estados Unidos: Princeton University.

Uppal, Y. (2009). The Disadvantaged Incumbents: Estimating Incumbency Effects in Indean States Legislatures. Public Choice, 138(1-2), 9-27, doi: 10.1007/s11127-008-9336-4

Veiga, L. G. y Veiga, F. J. (2007). Does opportunism pay off? Economics Letters, 96(2), 177-182, doi: 10.1016/j.econlet.2006.12.026

Vergara, R. (1997). El redescubrimiento de las instituciones: de la teoría organizacional a la ciencia política. En J. G. March y J. Olsen (eds.), El redescubrimiento de las instituciones. La base organizativa de la politica (pp. 9-40). Ciudad de México, México: Fondo de Cultura Económica.

Vergara, V. (2014). Desempeño en el cargo, ideología y factores personales como determinantes de la reelección municipal en 2008 (tesis para optar al grado de Magíster en Economía de Recursos Naturales y del Medio Ambiente). Dpto. de Economía, Facultad de Ciencia Económicas y Administrativas. Universidad de Concepción. Concepción, Chile.

Weyland, K. (2001). ¿La paradoja del éxito? Los determinantes del apoyo político al presidente Fujimori. Debates en Sociología, 25, 213-244.

Zavaleta, M. (2013). Las fuerzas gravitacionales de la descentralización: historia de tres elecciones subnacionales. Argumentos, 7(2), 17-25. 